\title{
Multi-polar Structures in Young Planetary and Protoplanetary Nebulae
}

\author{
Raghvendra Sahai \\ JPL, California Institute of Technology, Pasadena, CA 91109
}

\begin{abstract}
The imaging of young planetary and proto-planetary nebulae (PNe and PPNe) with unprecedented high angular resolution and dynamic range, using the Hubble Space Telescope, has led to the realisation that almost all of these objects are highly aspherical, with complex multipolar morphologies. The complexity, organization and symmetry of the morphological structures we find is forcing radical changes in, and inspiring fresh theoretical efforts to advance, our understanding of the mass-loss processes during late stellar evolution. In this paper, we review the HST data, and show some of the highlights of our imaging studies. Although the origins of many of the morphological features remains puzzling, we find that the general presence of multipolar structures support a model for PN formation in which the primary agent for shaping PNe are high-speed collimated outflows or jets which operate during the late AGB and/or early post-AGB evolutionary phase, and undergo episodic changes in their orientation (or collimated outflows operate quasi-simultaneously with different orientations).
\end{abstract}

\section{Introduction}

The extended circumstellar envelopes (CSEs) of most AGB stars appear largely spherically symmetric, and "filled" (i.e., not shell-like) (e.g., Bowers et al. 1983, Sahai \& Bieging 1993, Neri et al. 1998). However, drastic changes in the massloss geometry are observed during post-AGB evolution - planetary nebulae show bright rims and well defined shell-like structures, and a dazzling variety of morphologies, ranging from simple round and elliptical shapes, to much more complicated bipolar ones (e.g. Balick 1987, Schwarz, Corradi \& Melnick 1992). The challenge before us, therefore, is to determine the physical mechanisms responsible for the dramatic transformation in the symmetry of the ejected matter as the central star evolves from the AGB to a PN nucleus (i.e., a white dwarf).

Until recently, this challenge was believed to have been met, apparently rather successfully, by the Generalised Interacting Stellar Winds (GISW) model. In this model, the bright rims seen in PNe are shells of swept-up matter resulting from a fast $\left(~ \gtrsim 1000 \mathrm{~km} \mathrm{~s}^{-1}\right)$ stellar wind interacting with the slowly expanding CSE of the progenitor AGB star (Kwok 1982) and the asphericity of PNe is due to the presence of an equatorial density enhancement in the AGB CSE, which restricts the expansion of the swept-up shell at low latitudes (Balick 1987). Detailed hydrodynamic calculations (e.g., Soker and Livio 1989; Icke, Balick and Frank 1992; Frank et al. 1993) showed that a wide variety of axisymmetric shapes could be produced with the GISW model. However, it has not been able 
to satisfactorily address the following two issues - (i) the presence of a few $\mathrm{PNe}$ with point-symmetric structures (including those with ansae and "quadrupolar" PNe (e.g., Corradi \& Schwarz 1995, Manchado, Stanghellini, \& Guerrero 1996)) and (ii) the nature and origin of the asymmetry in the AGB envelopes.

An additional problem has now arisen for the GISW model with increasing observational evidence for the presence of fast $\left(\sim 100 \mathrm{~km} \mathrm{~s}^{-1}\right)$ bipolar outflows in PPNe (objects in transition between the PN and AGB phases, for which the central star is not yet hot enough to substantially ionise the circumstellar matter) and a few late AGB stars. These data imply that the mechanisms that shape $\mathrm{PNe}$ become operational before the PN phase.

Hence, observations of young PNe \& PPNe are of paramount importance to our understanding of PN formation. But, young objects are compact, and important structural details are usually blurred beyond recognition in groundbased optical images. Although radio interferometers enable imaging with high resolution $(\sim 0$ ". 1$)$, the images of PNe obtained, e.g. with the Very Large Array (e.g. Aaquist \& Kwok 1990) have insufficient dynamic range. In contrast, HST's stable PSF and high angular resolution has enabled the imaging of reflection or emission-line nebulosity with very high dynamic range, leading to a huge advance in our knowledge of the morphologies of young PNe and PPNe.

\section{HST OBSERVATIONS}

High-resolution imaging of young PNe and PPNe has revealed that the majority of these objects are characterised by multipolar lobes or bubbles, often distributed roughly point-symmetrically around the central star (e.g. Sahai \& Trauger 1998, Sahai 2000). We define multipolar objects as those which contain two or more distinct geometrical structures (e.g. lobes, ansae, rings, torii or disks) without coincidental geometrical axes, as well as objects with which show an overall S-shaped or otherwise point-symmetric morphology. In order to explain the origin of such morphologies, Sahai \& Trauger (1998) proposed that episodic high-speed jet-like outflows, operating during the protoplanetary or very late-AGB phase, are the primary agent for shaping PNe. We review below observations of multipolar morphology in PPNe and young PNe.

\subsection{Multipolar Protoplanetary Nebulae}

Detailed imaging of the reflection nebulosities in many PPNe has been carried out with HST Almost all PPNe imaged by us, e.g. (1) CRL 2688 or Egg Nebula (Sahai et al. 1998a,b), (2) IRAS 16342-3814, Roberts 22 \& Hen 3-401 (Sahai et al. 1999a,b,c), (3) IRAS 04296+3429 (Sahai 1999) and (4) IRAS 09371+1212 or Frosty Leo Nebula (Sahai et al. 2000), or other groups (e.g. IRAS 17150-3224 - Kwok et al. (1998); IRAS 16594-4656 \& 17245-3951 - Hrivnak et al. (1999); IRAS20028+3910 - Hrivnak et al. (2001); IRAS 17106-3046 - Kwok, Hrivnak \& Su (2000); IRAS 22272+5435, 17423-1755, 08005-2356, \& 06530-0213 - Ueta, Meixner \& Bobrowsky (2000); He 3-1475 - Borkowski, Blondin \& Harrington (1997) show bipolar or multipolar morphologies.

Such high angular resolution observations probing the kinematics of the circumstellar material are scarce. In the few objects for which data exist, there is evidence for multi-directional and/or high-speed outflows. The best-resolved 


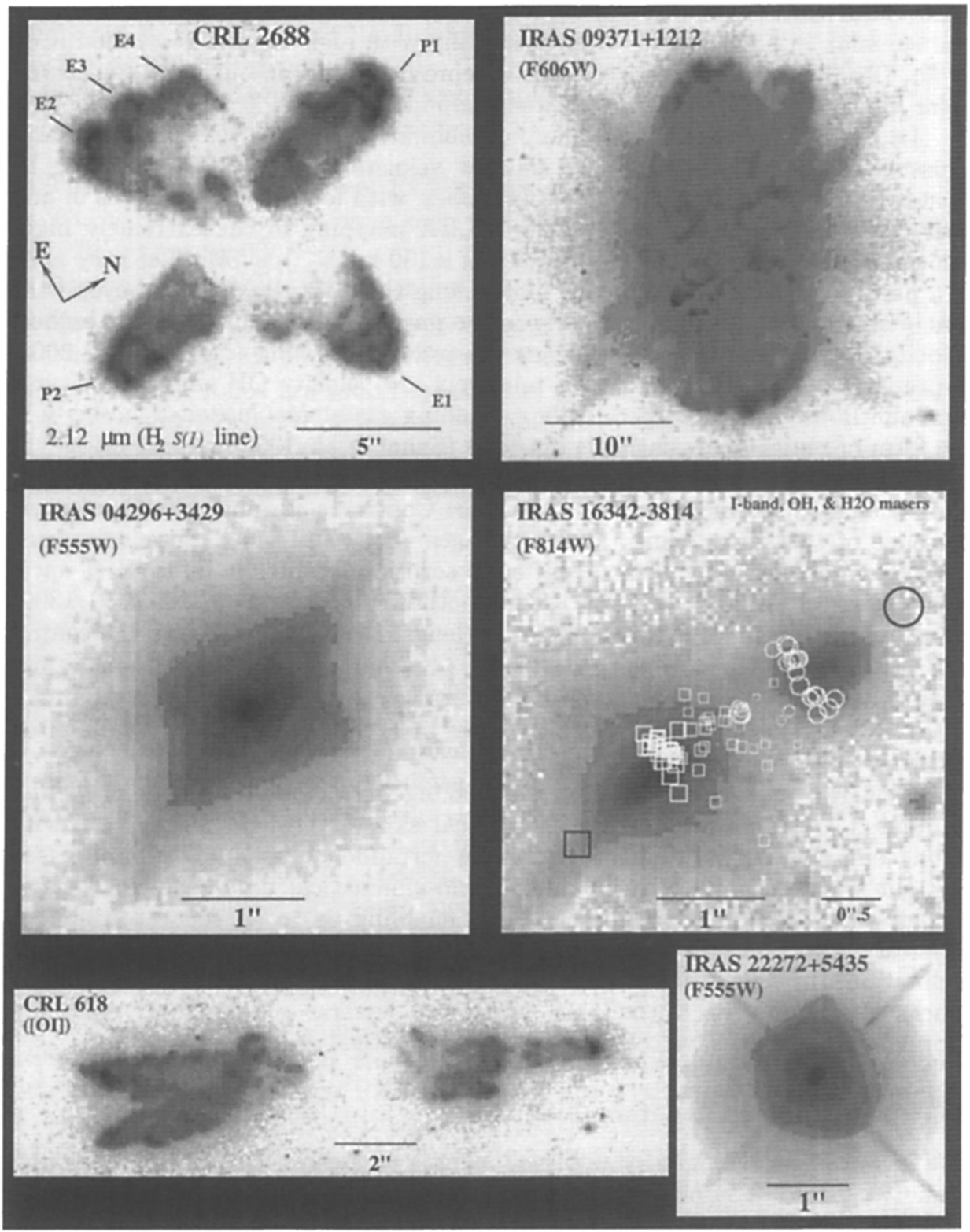

Figure 1. HST/WFPC2 images (in reverse grey-scale) of selected proto-planetary nebulae. In CRL 2688 , the extended outer regions of the nebula which represent the spherical envelope due to AGB massloss, are not shown. Note the wide variety of multipolar morphologies. 
PPNe, CRL 2688 and Frosty Leo (Fig. 1), show not only polar outflows, but also collimated features in or near their waist regions which may be attributed to the presence of multiple low-latitude jet-like outflows. By mapping the $\mathrm{CO} \mathrm{J}=2-1$ line emission in CRL 2688 interferometrically with high angular resolution, Cox et al. (2000) show that these features represent multiple outflows - even the polar lobes consist of multiple outflow components.

In IRAS16342-3814, VLA mapping shows the highest-velocity $\mathrm{OH}$ maser emission features (with projected outflow velocities up to $\sim 70 \mathrm{~km} \mathrm{~s}^{-1}$ ) to be clustered around the base of the optical lobes, with low velocity features in and near the waist (Sahai et al. 1999a). VLBA mapping of the extremely highvelocity $\mathrm{H}_{2} \mathrm{O}$ masers (projected velocity of $\pm 130 \mathrm{~km} \mathrm{~s}^{-1}$ ), shows that they arise in a pair of diamatrically opposed blobs along the optical axis, just beyond the tips of the optical lobes, presumably in the post-shock region where the highest velocity portion of a polar jet strikes the prior AGB wind (Sahai et al. 2002, in preparation). Roberts 22 shows relatively low-velocity $\mathrm{OH}$ emission features dominantly associated with radially expanding gas at low latitudes, possibly in the form of multiple low-latitude outflows (Sahai et al. 1999b).

In He 3-1475, characterised by a remarkable S-shaped chain of low-excitation, high-velocity blobs of shocked gas, Sánchez Contreras \& Sahai (2001) infer the presence of two very young (kinematic ages $\sim$ few $\times 10 \mathrm{yr}$ ) high velocity outflows, from the presence of blue-shifted absorption features in STIS/HST spectra. The faster (slower) of the two shows outflow velocities of $\sim 2300(150-1300)$ $\mathrm{km} \mathrm{s}^{-1}$. The former is highly collimated (length/width $\gtrsim 7$ ) close to the central star $\left(\sim 10^{16} \mathrm{~cm}\right)$ along a direction different from previous mass-ejection axes and shows a radially increasing velocity. He 3-1475 is thus one of the best examples of a PPN whose morphology is being shaped by high-speed post-AGB collimated outflows with changing directionality.

CRL 618, an evolved PPN (or very young PN) shows a number of highlycollimated lobes clustered along the optical axis of the nebula (Fig. 1), with strong emission in $\mathrm{H} \alpha$ and low-excitation forbidden lines. Our ground-based long-slit spectroscopy shows distinct spatio-kinematical differences in the forbidden and recombination line emission, enabling us to (i) separate different physical components in the nebula, (ii) revise earlier estimates of the nebular inclination, (iii) infer the shock-velocities $\left(75-200 \mathrm{~km} \mathrm{~s}^{-1}\right.$ ) associated with bowshocks seen at the tips of the lobes (Sánchez Contreras \& Sahai 2002), and (iv) show that the lobes have roughly similar kinematical ages.

\subsection{Multipolar Planetary Nebulae}

The largest, morphologically unbiased sample of images for young PNe comes from two SNAPshot observing programs on HST (GO 6353 and 8345: P.I. Sahai). The sample for these surveys was inspired by the class of very-lowexcitation objects discovered in an objective prism emission-line survey by Sanduleak \& Stephenson $(1972,1973)$ with very weak or negligible [OIII] emission. We therefore compiled our sample from the Acker et al. (1992) PNe catalog, selecting for (a) a relatively large $\mathrm{H} \alpha$-to-[O III] flux ratio $(>1.5)$, (b) an average $\mathrm{H} \alpha$ intensity $\gtrsim 6 \times 10^{-13} \mathrm{erg} \mathrm{s}^{-1} \mathrm{~cm}^{-2}$ arcsec $^{-2}$. (c) an optical or radio diameter (whichever was larger) $\geq 1$ arcsec. The $\mathrm{H} \alpha /[\mathrm{O}$ III] flux ratio is expected to be large in young PNe since (i) the relatively low stellar $\mathrm{T}_{\text {eff }}(\sim 25000-40000 \mathrm{~K})$ 
results in a low nebular excitation state (ii) the nebulae are compact with larger dust optical depths towards their central regions, resulting in a larger selective extinction of the shorter wavelength, more centrally concentrated [O III] line compared to $\mathrm{H} \alpha$. Three of the brightest PNe which meet our selection criteria - BD +30³639 (Harrington et al. 1997), MyCn 18 (Sahai et al. 1999d), and M 1-92 (Bujarrabal et al. 1998) - had already been studied in detail using HST.

The most notable conclusions from our HST observations are:

- There are no round PNe or PPNe; most objects are multipolar

- The variety of morphologies seen is much larger than known from groundbased surveys

- Point-symmetry is widely manifest

- Many PNe have internal geometric components (e.g. rings, inner hourglass)

- The central star is often offset from the centers of these components

- Faint round halos are found around the central aspherical nebula in many $\mathrm{PNe}$ and PPNe, (evidence for isotropic mass-loss on the AGB)

- Multiple concentric rings are found in the halo in several objects (evidence for episodic changes in the AGB mass-loss rate)

\section{A NEW MODEL FOR PRODUCING ASPHERICAL PLANE- TARY NEBULAE}

Our HST images of young PNe strongly suggest that the key to understanding the shaping of aspherical PNe is a physical mechanism which can generate multipolar and point-symmetric structures. Long-slit spectroscopic imaging of several bright PNe shows that point-symmetric features in the images are also kinematically point-symmetric (e.g. Guerrero, Vazquez, \& Lopez 1999). Bipolar rotating episodic jets (BRETs) have been invoked for creating point-symmetric structures in individual PNe (Lopez, Meaburn \& Palmer 1993). We (Sahai \& Trauger 1998) propose a two-step mechanism in which, first, a high-speed (few $100 \mathrm{~km} \mathrm{~s}^{-1}$ ) collimated or jet-like outflow (or outflows) operating during the late-AGB or early post-AGB evolutionary phase carve an imprint within an intrinsically spherical AGB mass-loss envelope. This imprint sets the stage for the development of an aspherical planetary nebula. Subsequent expansion of a hot, tenuous, even faster stellar wind inside this imprinted AGB envelope, then produces the observed PN, whose shape and structure depends in detail on how the characteristics (direction, strength, opening angle) of the jet-like outflows change with time. Extended dense equatorial structures, if present, also provide an impediment to the expansion of the fast wind in the equatorial plane. However, all of our PNe show complex aspherical shapes, regardless of whether or not such structures are visible in the images, which suggests that the equatorial structures are not the primary agent for producing aspherical PNe. Departures from point-symmetric structure can result in two ways: (1) departure from bipolar symmetry in the jets (which in the extreme would result in a one-sided jet), and (2) departures from symmetry in the progenitor AGB envelope. Such departures could lead to irregularly shaped nebular structures, which are also observed. 

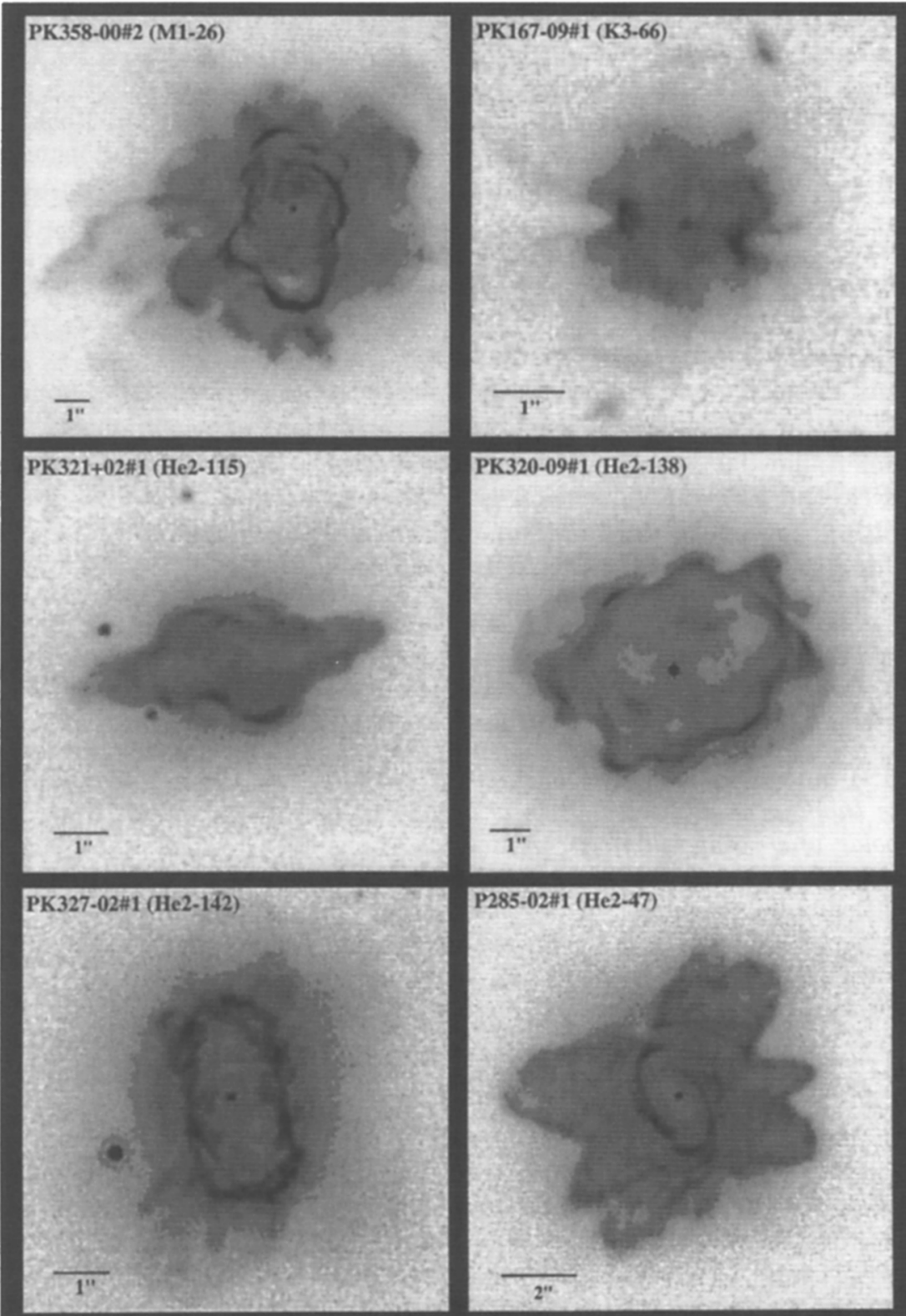

Figure 2. HST/WFPC2 $\mathrm{H} \alpha$ images (in reverse grey-scale) of young planetary nebulae from our survey. Note the wide variety of multipolar morphologies. Sharp features have been emphasized as described in Sahai \& Trauger (1998) 


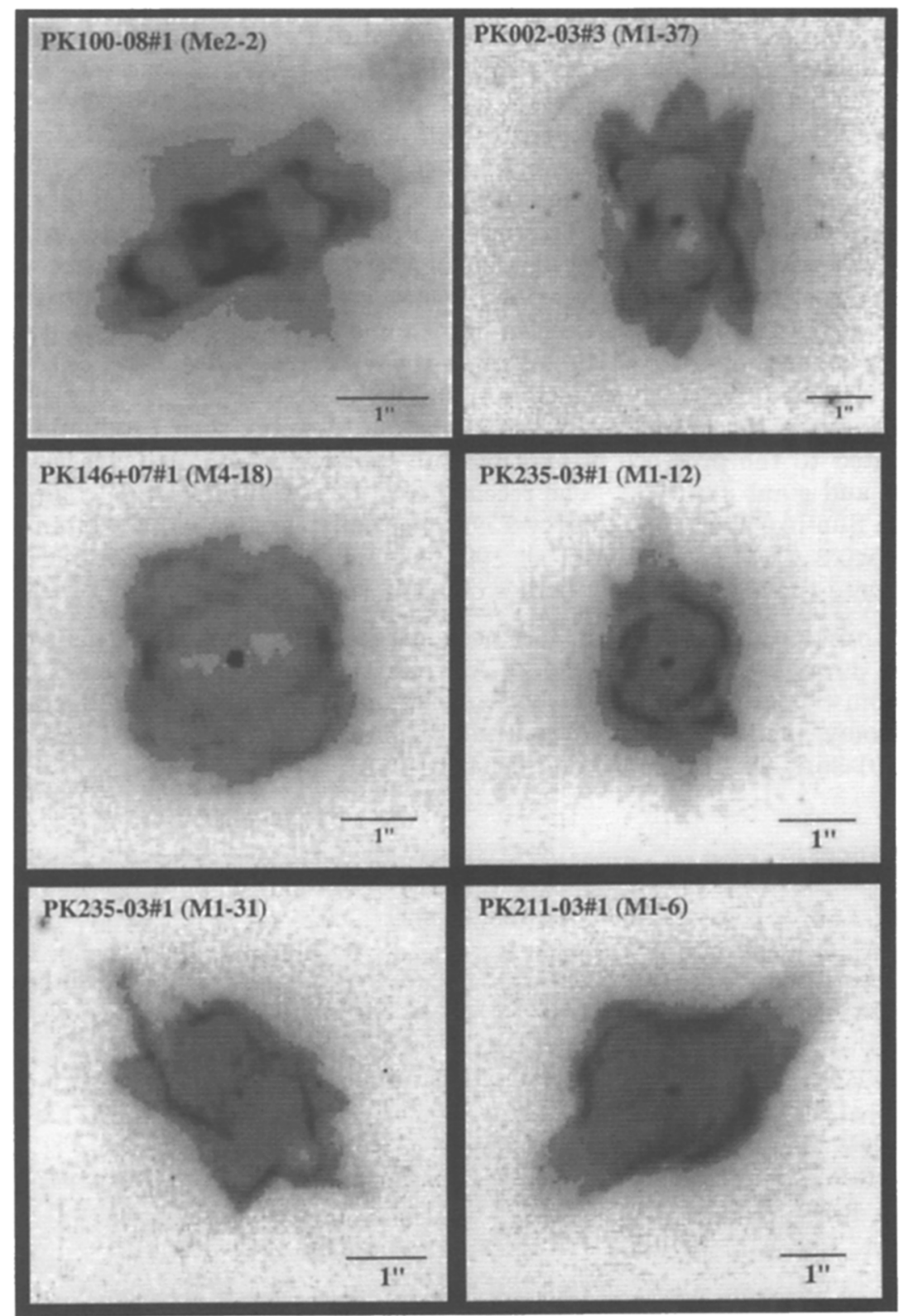

Figure 3. HST/WFPC2 $\mathrm{H} \alpha$ or [NII] 65586 images (in reverse greyscale) of young planetary nebulae. Note the wide variety of multipolar morphologies as in Fig. 2. Sharp features have been emphasized as described in Sahai \& Trauger (1998) 


\subsection{Mechanisms for Making Wobbling Jet-like Outflows}

Mechanisms for producing jet-like outflows, directly or indirectly, require the presence of a close binary companion. The most promising ones are those in which the outflows are (i) accretion disk-driven (e.g., Morris 1987; Soker 1996; Soker \& Livio 1994), or (ii) magneto-hydrodynamically collimated by a stellar toroidal magnetic field (e.g., Garcia-Segura 1997, Rozyczka \& Franco 1996). In case (i), the jet-axis may change due to a radiation-induced instability which can warp the accretion disk, causing it to precess or wobble (Livio \& Pringle 1997), whereas in case (ii), precession of the rotation axis of the star due to a binary companion can change the direction of the outflow. The variety and complexity of the structures seen in our sample of young PNe suggest that the changes in the properties of these outflows are episodic, and sometimes frequent, which may be difficult to achieve in a simple binary system. We suggest that the changes in the properties of these jets, and possibly their production, may be related to the presence of multiple sub-stellar companions (such as brown dwarfs and giant Jupiters). The recent detections of Jupiter-mass companions around Sun-like stars (e.g., Marcy 1998), specially those in close orbits (orbital radius $<0.2 \mathrm{AU}$ ) (e.g. Butler et al. 1997) should spur new theoretical efforts to investigate the affects of such bodies on the formation and shaping of PNe.

Acknowledgments. R.S. has been partially supported for this work by NASA through a Long Term Space Astrophysics grant (no. 399-20-61-00-00), and from STScI (operated by the Association of Universities for Research in Astronomy, Inc., under NASA contract NAS5-2655) GO grants 07839.01-96A, 07840.01-96A, 08307.01-97A, and 08345.01-97A.

\section{References}

Aaquist, O.B. \& Kwok, S. 1990, A\&ASS, 84, 229

Acker, A., Ochsenbein, F., Stenholm, B., Tylenda, R., Marcout, J. \& Schohn, S. 1992, Strasbourg-ESO Catalog of Galactic Planetary Nebulae, European Southern Observatory, Garching

Balick, B. 1987, AJ, 94, 671

Bowers, P.F., Johnston, K.J., \& Spencer, J.H. 1983, ApJ, 274, 733

Bujarrabal, V., Alcolea, J., Sahai, R., Zamorano, J., \& Zijlstra, A.A. 1998, A\&A, 331, 361

Butler, R.P., Marcy, G.W., Williams, E., Hauser, H., \& Shirts, P. 1997, ApJ, 474, L115

Corradi, R.L.M. \& Schwarz, H.E. 1995, A\&A, 293, 871

Frank, A., Balick, B., Mellema, G., \& Icke, V. 1993, ApJ, 404, L25

Garcia-Segura, G. 1997, ApJ, 489, L189

Guerrero, M.A., Vasquez, R., \& Lopez, J.A. 1999, AJ, 117, 967

Harrington, J.P., Lame, N.J., White, S.M., \& Borkowski, K.J. 1997, AJ, 113, 2147

Hrivnak, B.J., Kwok, S. \& Su, K.Y.L. 1999, ApJ, 524, 849

Icke, V., Balick, B., \& Frank, A. 1992, A\&A, 253, 524

Kwok, S. 1982, ApJ, 258, 280

Kwok, S., Su, K.Y.L. \& Hrivnak, B.J. 1998, ApJ, 501, L117

Latter, W.B., Dayal, A., Bieging, J.H., Meakin, C., Hora, J.L., Kelly, D.M. \& Tielens, A.G.G.M. 2000, ApJ, 539, 783 
Livio, M. \& Pringle, J. 1997, ApJ, 486, 835

Lopez, J.A., Meaburn, J., \& Palmer, J.W. 1993, ApJ, 415, L135

Manchado, A., Stanghellini, L., Guerrero, M.A. 1996, ApJ, 466, L95

Marcy, G.W. 1998, Nature, 391, 127

Morris, M. 1987, PASP, 99, 1115

Neri, R., Kahane, C., Lucas, R., Bujarrabal, V., \& Loup, C. 1998, A\&AS,130, 1

Rozyczka, M. \& Franco, J. 1996, ApJ, 469, L127

Sahai, R. 1999, ApJ, 524, L125

Sahai, R. 2000a, in Asymmetrical Planetary Nebulae II: From Origins to Microstructures, Eds. J.H. Kastner, N. Soker \& S.A. Rappaport, ASP, 199, 209

Sahai, R. 2000b, ApJ, 537, L43

Sahai, R. \& Bieging, J.H. 1993, AJ, 105, 595

Sahai, R. \& Trauger, J.T. 1998, AJ, 116, 1357

Sahai, R. et al. 1998a, ApJ, 492, L163

Sahai, R. et al. 1998b, ApJ, 493, 301

Sahai, R., Te Lintel Hekkert, P., Morris, M., Zijlstra, A. \& Likkel, L. 1999a, ApJ, 514, L115

Sahai, R., Bujarrabal, V. \& Zijlstra A. 1999b, ApJ, 518, L115

Sahai, R., Zijlstra, A., Bujarrabal, V., Te Lintel Hekkert, P. 1999c, AJ, 117, 1408

Sahai, R. et al. 1999d, AJ, 118, 468

Sahai, R. Bujarrabal, V., Castro-Carrizo, A. \& Zijlstra, A. 2000, A\&A, 360, L9

Sanduleak, N. \& Stephenson, C.B. 1973, ApJ, 185, 899

Sanduleak, N. \& Stephenson, C.B. 1972, ApJ, 178, 183

Schwarz, H.E., Corradi, R.J.M. \& Melnick, J. 1992, A\&AS, 96, 23

Soker, N. 1996, ApJ, 468, 774

Soker, N. \& Livio, M. 1994, ApJ, 421, 219

Ueta, T., Meixner, M. \& Bobrowsky, M. 2000, ApJ, 528, 861

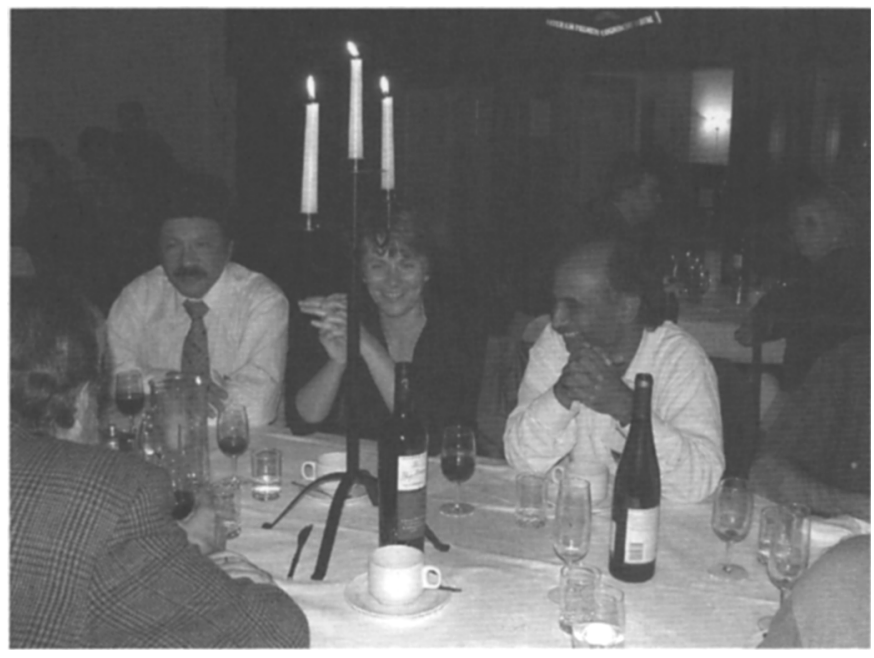

From left to right: Robert Rubin, Katrina Exter, Rahvendra Sahai 